\title{
Interfacial properties of natural magnetite particles compared with their synthetic analogue
}

\author{
E. Potapova ${ }^{\mathrm{a}, *}$, X. Yang ${ }^{\mathrm{b}}$, M. Westerstrand ${ }^{\mathrm{c}}$, M. Grahn $^{\mathrm{a}}$, A. Holmgren ${ }^{\mathrm{a}}$, J. Hedlund ${ }^{\mathrm{a}}$ \\ ${ }^{a}$ Chemical Technology, Luleå University of Technology, SE-971 87 Luleå, Sweden \\ ${ }^{\mathrm{b}}$ Research Centre for Eco-Environmental Sciences, Chinese Academy of Science, Beijing 100085, China \\ c Applied Geology, Luleå University of Technology, SE-971 87 Luleå, Sweden
}

\section{A R T I C L E I N F O}

Article history:

Available online 22 April 2012

\section{Keywords:}

Flotation reagents

Iron ores

Particle size

Surface modification

\begin{abstract}
A B S T R A C T
Understanding of the interactions between iron oxides and flotation reagents is important both for flotation and agglomeration of iron ore. Model systems comprising synthetic iron oxides and pure chemical reagents are commonly applied in experimental work in order to obtain high quality data and to ease the interpretation of the empirical data. Whether the results obtained using model systems are valid for iron ore minerals and commercial reagents is a question seldom addressed in the literature. It is shown in this work that previously reported results obtained from a model system, concerning adsorption of a carboxylate surfactant and sodium metasilicate onto synthetic magnetite nanoparticles, as obtained by in situ ATR-FTIR spectroscopy and contact angle measurements, are applicable to adsorption of flotation reagents on magnetite concentrate. Additionally, the problem of restoring magnetite wetting after flotation is addressed since good wetting of a magnetite concentrate is required to produce iron ore pellets by wet agglomeration. The results from the present work indicate that the wettability of both synthetic magnetite coated with surfactant and magnetite concentrate after flotation can be improved by adsorbing a hydrophilizing agent such as silicate or polyacrylate.
\end{abstract}

(c) 2012 Elsevier Ltd. All rights reserved.

\section{Introduction}

Interfacial phenomena such as adsorption at the solid/liquid interface and wetting are important for several mineral processing operations including desliming, flocculation, flotation and agglomeration. In situ infrared spectroscopy and contact angle measurements have proven to be a powerful combination of tools for studying adsorption on mineral surfaces and its effect on surface wettability (Fuerstenau, 2007). ATR-FTIR spectroscopy allows monitoring interactions in situ in the presence of water and provides information about adsorption kinetics and equilibrium as well as the surface complexes formed (Smart et al., 2007).

Since most of the minerals are not transparent for infrared radiation, ATR elements coated with thin films of mineral particles are commonly applied in the adsorption studies by ATR-FTIR spectroscopy (Smart et al., 2007). Natural mineral particles are not always appropriate for this type of measurements since they may have relatively low surface area resulting in a low signal-to-noise ratio and may contain impurities that would complicate the interpretation of the spectroscopic results. For that reason, synthetic mineral particles with high surface area are commonly applied in the adsorption studies by ATR-FTIR spectroscopy.

\footnotetext{
* Corresponding author. Tel.: +46 920 491776; fax: +46 920491199.

E-mail address: elisaveta.potapova@ltu.se (E. Potapova).
}

In our previous work (Potapova et al., 2010a,b), a method based on in situ ATR-FTIR spectroscopy was developed and successfully used for studying adsorption of flotation related chemicals from aqueous solutions on thin films of synthetic iron oxides. The spectroscopic data were later complemented by contact angle and zeta-potential measurements (Potapova et al., 2011).

The interactions between iron oxides and flotation reagents are important, not only for the performance in flotation of apatite from magnetite, but also for the subsequent agglomeration of magnetite concentrate to produce iron ore pellets. Reduced wetting of the magnetite concentrate due to adsorption of a flotation collector results in lower pellet strength (Forsmo et al., 2008; Gustafsson and Adolfsson, 1997; Iwasaki et al., 1967) and may hamper the production capacity of a pelletizing plant.

The scope of the present work was to characterise the interfacial properties of natural magnetite particles cleaned by magnetic separation and flotation and to compare with the properties of the synthetic magnetite nanoparticles used in our previous experimental work, especially to test whether the conclusions drawn regarding adsorption behaviour and wettability of synthetic magnetite are valid for mineral particles. Whereas this aspect is seldom addressed in the literature, the difference in interfacial properties of synthetic and natural materials is an important issue to consider when substituting synthetic particles for their mineral analogue in experimental work. 


\section{Materials and methods}

\subsection{Materials}

Magnetite nanocrystals were synthesized and purified according to the procedure described previously (Potapova et al., 2010b). A dispersion of magnetite in distilled water was further diluted with methanol and degassed distilled water resulting in a working dispersion containing 25 vol.\% methanol and ca $1.1 \mathrm{mg} \mathrm{mL}^{-1}$ magnetite. The dispersion was stored in a refrigerator in order to minimise oxidation of magnetite.

Magnetite pellet concentrate after flotation and magnetic separation from the pelletizing plant in Kiruna, Sweden was provided by LKAB. The concentrate had been stored at ambient conditions for more than 2 years. Moist when received, the concentrate was stored in a refrigerator in a sealed plastic bag. Prior to usage, the concentrate was dried in an oven at $50{ }^{\circ} \mathrm{C}$.

Flotation collector Atrac 1563 (Akzo Nobel) and dispersant/ depressant water glass were provided by LKAB, Sweden.

Water glass is an aqueous solution of sodium silicate, in this case with a $\mathrm{SiO}_{2}: \mathrm{Na}_{2} \mathrm{O}$ weight ratio of 3.25 . Sodium metasilicate $\left(\mathrm{Na}_{2}\right.$ $\mathrm{SiO}_{3} \cdot 9 \mathrm{H}_{2} \mathrm{O}, \geqslant 98 \%$, Sigma) was used in the experiments with synthetic magnetite as an analytical grade alternative of water glass.

Flotation collector Atrac 1563 has a complex chemical composition: $50-100 \%$ ethoxylated tall oil ester of maleic acid, and 15\% maleic anhydride (Akzo Nobel material safety data sheet). Since exact composition and chemical structure of Atrac 1563 were not specified by the supplier, dodecyloxyethoxyethoxyethoxyethyl maleate (Sigma-Aldrich) was used as a model flotation collector to ease the interpretation of the spectroscopic data. Fig. 1 shows the supposed chemical structure of flotation collector Atrac 1563 and the structure of the surfactant used as a model flotation collector.

Calcium chloride $\left(\mathrm{CaCl}_{2} \cdot 2 \mathrm{H}_{2} \mathrm{O}, 95 \%\right.$, Riedel-de Haen) was used to provide a solution with calcium ions. Sodium chloride $(\mathrm{NaCl}$, pro analysis, Riedel-de Haen) at a concentration of $10 \mathrm{mM}$ was used as ionic medium in spectroscopic, zeta-potential and contact angle measurements, if not stated otherwise.

Dispex A40 and Dispex N40 (ammonium and sodium polyacrylate, respectively, BASF) with the average molecular weight of 4000 were used for surface modification of magnetite after surfactant adsorption.

All aqueous solutions were prepared using distilled water. Distilled water for the spectroscopic and contact angle experiments was degassed under vacuum to minimise the amount of dissolved gases. The $\mathrm{pH}$ of the working solutions was adjusted using aqueous solutions of sodium hydroxide ( $\mathrm{NaOH}$, pro analysi, Merck) and hydrochloric acid ( $\mathrm{HCl}, 37 \%$, pro analysi,Merck).

\subsection{Methods}

2.2.1. Characterisation of the synthetic and natural magnetite particles 2.2.1.1. X-ray diffraction (XRD). XRD patterns of both synthetic and natural magnetite particles were collected with a Siemens D5000

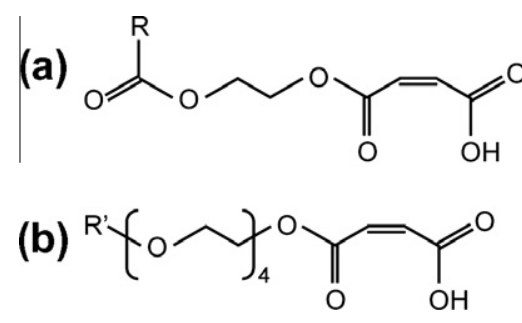

Fig. 1. Supposed chemical structure of Atrac 1563 (a) and the structure of dodecyloxyethoxyethoxyethoxyethyl maleate (b). $\mathrm{R}$ represents the alkyl chain in fatty acids, $\mathrm{R}^{\prime}$ represents the linear alkyl chain $\mathrm{CH}_{3}\left(\mathrm{CH}_{2}\right)_{11}$. diffractometer running in Bragg-Brentano geometry using $\mathrm{Cu} \mathrm{K} \alpha$ radiation.

2.2.1.2. High-resolution scanning electron microscopy (HR-SEM). The morphology of synthetic and natural magnetite particles without any coating was investigated with an FEI Magellan 400 field emission extreme high resolution scanning electron microscope (XHRSEM) using an accelerating voltage of $1 \mathrm{kV}$.

2.2.1.3. Energy dispersive X-ray spectroscopy (EDS). Chemical analysis of the natural magnetite particles in the size range from 0.22 to $8 \mu \mathrm{m}$ was carried out by SEM-EDS using an Oxford instruments XMax50 SDD detector, combined with a Zeiss Merlin field emission SEM. In the EDS measurements, the accelerating voltage of $20 \mathrm{kV}$ was applied.

2.2.1.4. BET surface area. Specific surface area of synthetic magnetite particles was estimated using the BET method from nitrogen adsorption data recorded at liquid nitrogen temperature using a Micrometrics ASAP 2010 gas adsorption analyser. Degassing was performed by evacuating the sample at $130^{\circ} \mathrm{C}$ overnight.

2.2.1.5. Zeta-potential. The zeta-potential of both synthetic and natural magnetite at different $\mathrm{pH}$ was determined by electrophoresis using a ZetaCompact instrument equipped with a charge-coupled device (CCD) tracking camera. The electrophoretic mobility data was further processed by the Zeta4 software applying the Smoluchowski equation. The zeta-potential was calculated as an average of three replicates.

The natural magnetite sample for the zeta-potential measurements was collected in the form of a slurry after flotation at the LKAB concentration plant in Kiruna, Sweden. Due to the particle size limitations of the measuring technique, the zeta-potential was measured using the particles with a size of $0.2-8 \mu \mathrm{m}$, obtained by vacuum filtration of the slurry using cellulose filters. Prior to filtration, the filters were washed with a $5 \%$ aqueous solution of acetic acid (Odman et al., 1999).

Process water used in the zeta-potential measurements on the synthetic magnetite was obtained from the filtration step at the LKAB concentration plant in Kiruna, Sweden. The process water was filtered through a cellulose filter (Millipore, $0.22 \mu \mathrm{m}$ pore size), washed with a $5 \%$ aqueous solution of acetic acid (Odman et al., 1999). The concentration of dissolved species in the process water was analysed at ALS Scandinavia in Luleå, Sweden, accredited according to the international standards ISO 17025, ISO 9001:2000, SS EN 1484 and ISO/IEC Guide 25. The samples were analysed using inductively coupled plasma optical emission spectrometry (ICP-OES) and inductively coupled plasma sector field mass spectrometry (ICP-SFMS). Total organic carbon (TOC) was analysed using a Shimadzu TOC-5000 high-temperature combustion instrument.

2.2.1.6. Contact angle. The static sessile drop method was used to determine the contact angle of the synthetic magnetite nanoparticles. A thin film of synthetic magnetite was produced on a substrate by spreading $0.5 \mathrm{~mL}$ of the magnetite dispersion described in Section 2.1 and drying it in air at room temperature. Contact angle measurements were performed using a Fibro 1121/1122 DAT - Dynamic Absorption and Contact Angle Tester equipped with a CCD camera. The measurement was performed by placing a $4 \mu \mathrm{L}$ water droplet onto the magnetite coated substrate using a microsyringe. A series of images of the droplet were recorded and analysed using the DAT 3.6 software. For each film, the measurement was repeated 8-10 times by applying fresh droplets. The value of the contact angle was determined as an average of the replicates. Consecutive adsorption of $\mathrm{CaCl}_{2}, \mathrm{Na}_{2} \mathrm{SiO}_{3}$, collector, 
and a hydrophilizing agent (either ammonium polyacrylate for $1 \mathrm{~h}$ or sodium metasilicate for $24 \mathrm{~h}$ ) was performed on the magnetite film at the same concentrations and $\mathrm{pH}$ as in the spectroscopic measurements. After each adsorption step, the contact angle of the magnetite film was measured. Further experimental details are described elsewhere (Potapova et al., 2012).

The advancing contact angle of the natural magnetite particles was determined by the Washburn method using a Krüss K100 force tensiometer. Liquid sorption by the magnetite powder was recorded as a function of immersion time and Krüss LabDesk 3.1 software was used to calculate the advancing contact angle applying the Washburn equation. First, the capillary constant of the Washburn equation was estimated for each sample using $n$-hexane. Thereafter, the contact angle of the magnetite powder was measured using deionized water. The values of the capillary constant and the contact angle were calculated as an average of three replicates. For a single measurement, ca. $1 \mathrm{~g}$ of the magnetite powder was used.

To investigate the effect of different reagents on the wettability of the natural magnetite particles, batch adsorption was performed using suspensions containing $10 \mathrm{~g}$ magnetite per ca. $40 \mathrm{~mL}$ solution at $\mathrm{pH} 9$ and room temperature. After adsorption, the solution was decanted and the magnetite was dried in an oven overnight at $50{ }^{\circ} \mathrm{C}$.

To investigate the effect of calcium ions and water glass on collector adsorption onto the natural magnetite particles, magnetite powder was preconditioned for $1 \mathrm{~h}$ in an aqueous solution containing water glass at a concentration of $1.0 \mathrm{mg} \mathrm{g}^{-1}$ magnetite (or ca. $1.1 \mathrm{mM}$ [Si]), together with $10 \mathrm{mM} \mathrm{NaCl}$ or $4 \mathrm{mM} \mathrm{CaCl}_{2}$. Thereafter, the flotation collector Atrac 1563 was added to the suspension and was allowed to adsorb for $20 \mathrm{~min}$.

To investigate the effect of sodium polyacrylate and water glass on the wettability of flotated magnetite concentrate, as-received magnetite concentrate was treated with these reagents for $1 \mathrm{~h}$ and $9 \mathrm{~h}$, respectively. The effect of sodium polyacrylate was examined in the aqueous solutions of either calcium chloride or sodium chloride.

\subsubsection{In situ ATR-FTIR spectroscopy}

Infrared spectra were recorded using a Bruker IFS 66v/S spectrometer equipped with a deuterated triglycine sulphate (DTGS) detector and a liquid nitrogen cooled mercury-cadmium-telluride (MCT) detector. Magnetite coated ZnSe ATR crystals (Crystran Ltd.) in the form of a trapeze with $45^{\circ}$ cut edges and dimensions of $50 \times 20 \times 2 \mathrm{~mm}$ were used in this study.

The incidence angle of the infrared beam was set to $45^{\circ}$. Both single beam background and sample spectra were obtained by averaging 500 scans at a resolution of $4 \mathrm{~cm}^{-1}$. Data processing was performed using the Bruker Opus 4.2 software. All the spectroscopic experiments were performed on solutions at $\mathrm{pH} 8.5$ and at room temperature. The $\mathrm{pH}$ of the solution was controlled by a Mettler Toledo T70 titrator. Further experimental details are described elsewhere (Potapova et al., 2010b; Yang et al., 2008).

\section{Results and discussion}

\subsection{Characterisation of the synthetic and natural magnetite particles}

XRD patterns presented in Fig. 2 indicate pure crystalline magnetite without any other phases present in amounts detectable by XRD. Broader reflections in Fig. 2a compared to the ones in Fig. 2b indicate that the crystal size was much smaller for the case of the synthetic magnetite than for the magnetite mineral, in concert with SEM observations, see below.

Fig. 3 shows HR-SEM images of the cross-section of a thin layer of synthetic magnetite nanoparticles deposited on a ZnSe substrate and natural magnetite particles spread over a carbon tape.

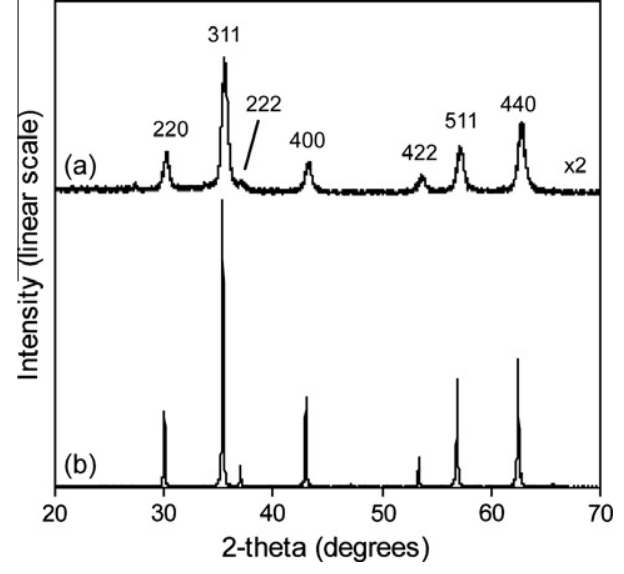

Fig. 2. XRD patterns of the synthetic (a) and natural (b) magnetite crystals. The reflections originating from magnetite are indexed by the appropriate Miller indices. For the sake of clarity, the intensity of pattern (a) was multiplied by a factor 2 and shifted.

Synthetic magnetite particles (Fig. 3a) showed a spherical habit with a diameter of 5-15 $\mathrm{nm}$. The particles were partially aggregated and formed a continuous porous film on the ZnSe substrate. The particles in the magnetite concentrate (Fig. 3b) had irregular shape and varied in size. The coarse magnetite particles in Fig 3b were covered by very fine particles (less than $1 \mu \mathrm{m}$ in size), some of which, according to the EDS results, had a high content of silicon and aluminium and could be the remains of aluminosilicates present in the iron ore before concentration.

The specific surface area of synthetic magnetite determined from nitrogen adsorption data was about $90 \mathrm{~m}^{2} \mathrm{~g}^{-1}$, which is much
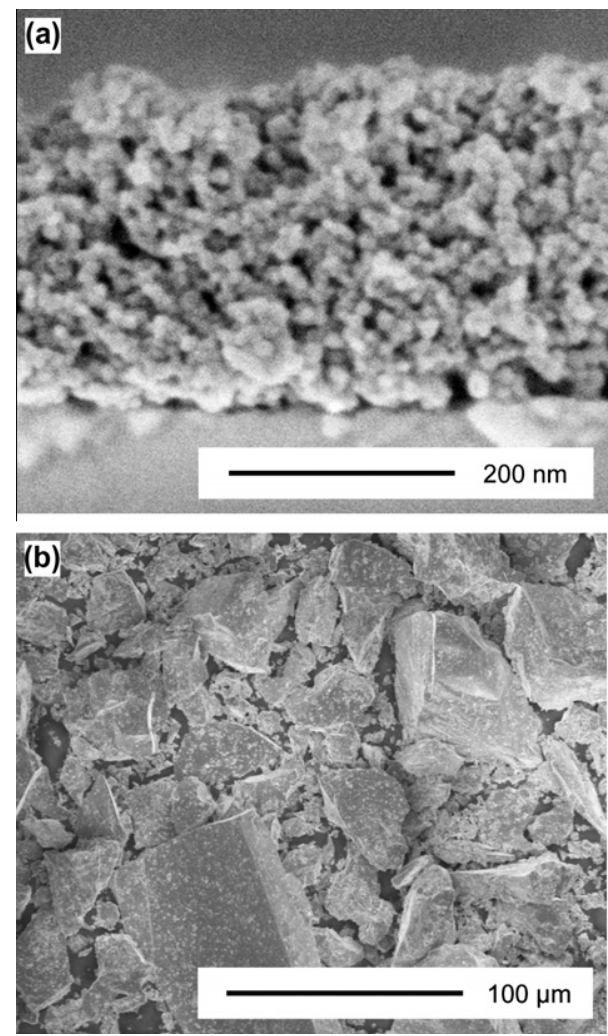

Fig. 3. HR-SEM images of synthetic magnetite particles on a ZnSe substrate (a) and natural magnetite particles on a carbon tape (b) 
higher than the specific surface area of natural magnetite that is only about $0.5 \mathrm{~m}^{2} \mathrm{~g}^{-1}$ (Forsmo, 2005). The synthetic magnetite thus had much more surface sites per gram material available for adsorption, which motivates the use of synthetic magnetite in model systems.

Regarding the contact angle of synthetic and natural magnetite particles, different measuring techniques had to be used due to the difference in the particles' size. The static sessile drop method is suitable for measuring the contact angle of colloid particles (like synthetic magnetite nanoparticles used in this work), providing that a closely packed layer of particles can be formed (Shang et al., 2008). Using this method, the contact angle of synthetic magnetite in the present work was determined to be $15-25^{\circ}$. A value of $12 \pm 1^{\circ}$ was previously reported (Galindo-González et al., 2005) for spherical magnetite nanoparticles with a mean size of $11 \pm 2 \mathrm{~nm}$. Wang and Ren (2005) measured the contact angle of water on a polished surface of natural magnetite using the static sessile drop method and reported a value of $25 \pm 5^{\circ}$. The contact angle determined in the present work is thus comparable to previously reported contact angles.

The contact angle of natural mineral powders is commonly estimated by the Washburn method. In this method, packing of particles is also important since it may affect the penetration rate of the wetting liquid and thus the measured value of the contact angle Kirchberg et al., 2011).

For the natural magnetite particles used in this work, the contact angle was estimated to be $50-60^{\circ}$, which is similar to previously reported values. A contact angle of $72-75^{\circ}$ (Kirchberg et al., 2011) was reported for unsieved magnetite powder with a particle size of $\leqslant 146 \mu \mathrm{m}$ and irregular particle shape. For the magnetite with a size of $86 \%-74 \mu \mathrm{m}$, the contact angle was determined to be $46^{\circ}$ (Qiu et al., 2004). Thereby, the contact angle of natural magnetite measured by the Washburn method is normally reported to be higher than the contact angle of synthetic magnetite nanoparticles. This difference could be partly due to the measuring technique but also the particle size and impurities present on the surface of natural magnetite particles. Apart from that, the contact angle measured by the sessile drop method may vary depending on the surface roughness, resulting in an underestimated contact angle for hydrophilic surfaces and an overestimated contact angle for hydrophobic surfaces. For the case of natural magnetite used in this work, the high contact angle could also be caused by adsorbed flotation collector since the iron ore was concentrated both by magnetic separation and flotation. The amount of flotation collector adsorbed on the magnetite surface after flotation was previously estimated to be $10-30 \mathrm{~g} \mathrm{t}^{-1}$ (Forsmo et al., 2008). Already a partial coverage by a surfactant could be enough to obtain a hydrophobic surface (Holmberg et al., 2003).

Fig. 4 illustrates the zeta-potential of the synthetic and natural magnetite particles as a function of $\mathrm{pH}$. The zeta-potential curve for the synthetic magnetite in a $10 \mathrm{mM} \mathrm{NaCl}$ solution (empty triangles) exhibits the shape typically observed for iron oxides, with the IEP at pH 7 (Potapova et al., 2011).

However, the zeta potential of the $0.22-8 \mu \mathrm{m}$ particle fraction of the magnetite slurry in the process water after flotation (filled squares) showed a completely different dependency on $\mathrm{pH}$, with two IEP observed at about $\mathrm{pH} 11$ and at $\mathrm{pH} \leqslant 2$. Such behaviour could be due to the specific adsorption of soluble species present in the process water onto the surface of the particles and due to the presence of mineral impurities in the slurry.

To test the first hypothesis, the zeta-potential was measured on the synthetic magnetite particles dispersed in the process water from the LKAB concentrating plant in Kiruna, Sweden (open diamonds in Fig. 4).

The curves for the iron ore concentrate after flotation and the synthetic magnetite in the process water are fairly similar and characterised by a weakly negative zeta-potential almost in the

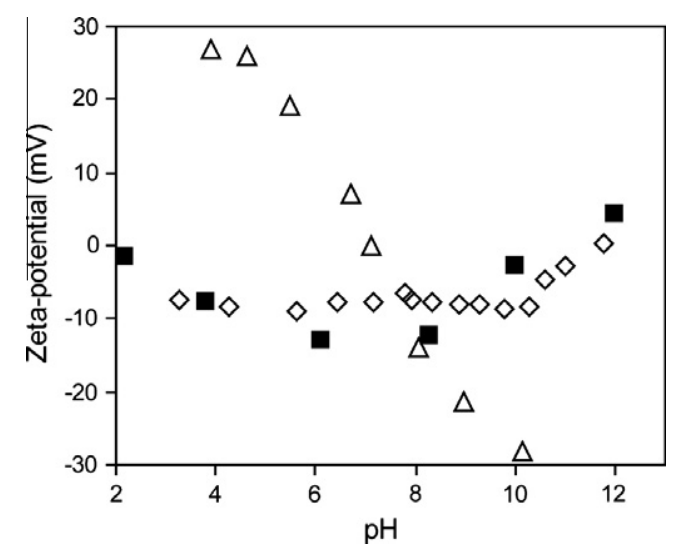

Fig. 4. Zeta-potential as a function of $\mathrm{pH}$; of the synthetic magnetite particles in $10 \mathrm{mM} \mathrm{NaCl}(\Delta)$ and in the process water from the LKAB concentrating plant in Kiruna, Sweden $(\diamond)$; of the $0.22-8 \mu \mathrm{m}$ fraction of the iron ore concentrate in the

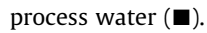

whole $\mathrm{pH}$ range with an increase in the zeta-potential at highly alkaline $\mathrm{pH}$. These results suggest that the dissolved species present in the process water can have a significant effect on the zeta-potential of magnetite, in concert with the results reported previously (Potapova et al., 2011). The measured concentration of dissolved species present in the process water is shown in Table 1.

The decrease of the zeta-potential of the magnetite particles in the process water at $\mathrm{pH}<8$ and the shift of the IEP to a lower $\mathrm{pH}$ as compared to the zeta-potential of the synthetic magnetite in $10 \mathrm{mM} \mathrm{NaCl}$ could be explained by the specific adsorption of anions such as sulphate, bicarbonate, and silicate on the magnetite surface. The specific adsorption of cations such as calcium and magnesium on the magnetite surface could result in the increase of the zeta-potential of the magnetite particles in the process water at $\mathrm{pH}>8$ and could cause a shift of the IEP to a higher $\mathrm{pH}$ as compared to the zeta-potential of the synthetic magnetite in $10 \mathrm{mM}$ $\mathrm{NaCl}$.

To determine whether the natural magnetite particles used in the zeta-potential measurements contained any mineral impurities, the particles of the $0.22-8 \mu \mathrm{m}$ fraction of the magnetite slurry obtained after flotation were investigated by SEM-EDS, which showed that the measured particle fraction contained mainly silicon, aluminium, and oxygen. Thereby, it was concluded that the mineral particles used in the zeta-potential measurements were to a large extent aluminosilicate. Whereas aluminosilicate minerals at the LKAB concentrating plant in Kiruna, Sweden, are removed from the iron ore by magnetic separation, small aluminosilicate particles still remain in the iron ore concentrate after magnetic separation.

\subsection{Adsorption behaviour of model compounds and commercial flotation reagents}

Careful analysis of the ATR-FTIR spectra of model compounds and commercial flotation reagents can provide important information about their chemical structure and adsorption mechanisms.

Table 1

Concentration of solutes in the process water.

\begin{tabular}{lllllllllll}
\hline Specie & $\mathrm{Ca}$ & $\mathrm{S}$ & $\mathrm{Na}$ & $\mathrm{Cl}$ & $\mathrm{K}$ & $\mathrm{NO}_{3}$ & $\mathrm{Mg}$ & $\mathrm{HCO}_{3}$ & $\mathrm{Si}$ & $\mathrm{TOC}$ \\
\hline $\begin{array}{c}\text { Concentration } \\
(\mathrm{mM})\end{array}$ & 8.8 & 10.6 & 9.5 & 9.0 & 2.4 & 2.0 & 1.6 & 1.1 & 0.5 & $4.3^{\mathrm{a}}$
\end{tabular}

a Concentration in $\mathrm{mg} \mathrm{L}^{-1}$. 
Fig. 5 shows spectra of the flotation collector Atrac 1563 and the model collector adsorbed on synthetic magnetite at $\mathrm{pH}$ 8.5. Atrac 1563 and the model collector display rather similar spectral features, in agreement with the structural resemblance of the head groups in these compounds (Fig. 1). A significant difference that should be pointed out is the absence of the band at $1104 \mathrm{~cm}^{-1}$ in the spectrum of Atrac 1563, associated with the stretching vibrations of the $\mathrm{C}-\mathrm{O}-\mathrm{C}$ groups $(v(\mathrm{C}-\mathrm{O}-\mathrm{C}))$ in the ethoxy-chains (Beentjes et al., 2006). This implies a low degree of ethoxylation of the molecules in Atrac 1563 as compared to the model collector, in concert with the chemical structures shown in Fig. 1.

In aqueous solutions at $\mathrm{pH} 8.5$, the carboxylic acid groups in Atrac 1563 and the maleic acid ester become deprotonated forming a negatively charged carboxylate ion as indicated by the presence of the symmetric $\left(v_{\mathrm{s}}\left(\mathrm{COO}^{-}\right)\right)$and asymmetric $\left(v_{\mathrm{as}}\left(\mathrm{COO}^{-}\right)\right)$ stretching vibrations bands (Dobson and McQuillan, 1999) in the spectra of these compounds. Previously, it was concluded that carboxylate ions were not likely to be responsible for collector adsorption on iron oxides at $\mathrm{pH} 8.5$ in the absence of a background electrolyte due to electrostatic repulsion (Potapova et al., 2010a) and that the adsorption took place via the non-charged polar ester carbonyl and ethoxy groups. However, the presence of a background electrolyte in the present work could reduce the repulsion between the ions and the magnetite surface further facilitating collector adsorption (Potapova et al., 2010b).

For the adsorption of maleic acid on hematite, two co-existing surface complexes were reported (Hwang and Lenhart, 2008) an inner-sphere complex characterised by the $v_{\mathrm{s}}\left(\mathrm{COO}^{-}\right)$band at ca $1407 \mathrm{~cm}^{-1}$, and an outer-sphere complex characterised by the $v_{\mathrm{s}}\left(\mathrm{COO}^{-}\right)$band at ca $1430 \mathrm{~cm}^{-1}$. Similarly, Atrac 1563 and the model collector both containing maleic acid in their head groups could form an outer-sphere complex and an inner-sphere complex on magnetite.

Additionally, ester carbonyls present in the collectors could contribute to their adsorption on the magnetite surface, as discussed elsewhere (Potapova et al., 2010a,b).

Thereby, despite the difference in the structure of the head groups, similar adsorption mechanism was observed for both collectors justifying the usage of the maleic acid ester as a model compound for studies of the adsorption behaviour of commercial collectors such as Atrac 1563.

Fig. 6 shows infrared spectra of sodium metasilicate and water glass adsorbed on magnetite at $\mathrm{pH}$ 8.5. Despite the fact that these soluble silicates have different $\mathrm{SiO}_{2}: \mathrm{Na}_{2} \mathrm{O}$ weight ratio, the spectral line shapes in Fig. 6 are rather similar.

At this $\mathrm{pH}$, the most pronounced band in the infrared spectrum is located at $1020 \mathrm{~cm}^{-1}$ with two shoulders at ca. $1120 \mathrm{~cm}^{-1}$ and

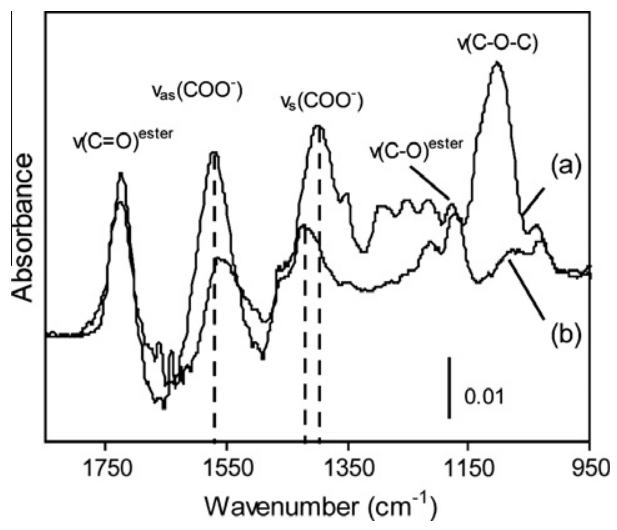

Fig. 5. ATR-FTIR spectra of maleic acid ester (a) and Atrac 1563 (b) adsorbed on magnetite for $6 \mathrm{~h}$ from $25 \mathrm{mg} \mathrm{L}^{-1}$ aqueous solutions at $\mathrm{pH} 8.5$ with $10 \mathrm{mM} \mathrm{NaCl}$ as background electrolyte. ca. $950 \mathrm{~cm}^{-1}$. The bands at $950 \mathrm{~cm}^{-1}$ and $1020 \mathrm{~cm}^{-1}$ originate from adsorbed monomeric and oligomeric silicate species, respectively, whereas the band at $1120 \mathrm{~cm}^{-1}$ is associated with the 3-dimentional silica framework structure (Yang et al., 2009).

Although the time of adsorption was higher for sodium metasilicate, greater absorption intensity is observed for water glass due to a larger amount of magnetite used in the adsorption experiment with water glass.

According to previous results (Yang et al., 2008), the speciation of the adsorbed silicate on the magnetite surface is determined by $\mathrm{pH}$ and silicate concentration rather than by the $\mathrm{SiO}_{2}: \mathrm{Na}_{2} \mathrm{O}$ ratio suggesting that silicate sources with different composition could be expected to show similar adsorption behaviour.

\subsection{Adsorption of flotation reagents and effect on magnetite surface properties}

The effect of calcium ions and sodium metasilicate on the adsorption of the model collector on synthetic magnetite was investigated in our previous work (Potapova et al., 2010b). It was concluded that sodium metasilicate could suppress the adsorption of the model collector on magnetite but only in the absence of calcium ions. When calcium ions were present in the system, the adsorption of the collector was dramatically increased both with and without sodium metasilicate present in solution.

This conclusion was confirmed by contact angle measurements (see Table 2) showing that whereas the contact angle decreased upon treatment with calcium and metasilicate, addition of the model collector resulted in a considerable increase in the contact angle. These results imply that sodium metasilicate could not prevent the adsorption of the model collector in the presence of calcium ions. Approximately the same increase in contact angle was observed for the model collector and Atrac 1563, confirming that these compounds had similar effect on the wettability of magnetite.

Further, it was investigated whether the conclusions regarding the effect of calcium and silicate on collector adsorption onto synthetic magnetite were applicable to natural magnetite particles. Adsorption of Atrac 1563 and water glass on magnetite concentrate was performed in the presence and absence of calcium ions and the contact angle of the concentrate was measured by the Washburn method, see Fig. 7.

In the absence of calcium ions, the contact angle did not change upon collector adsorption indicating that no or very little adsorption took place on magnetite concentrate pre-treated with water glass. However, collector adsorption in the presence of calcium

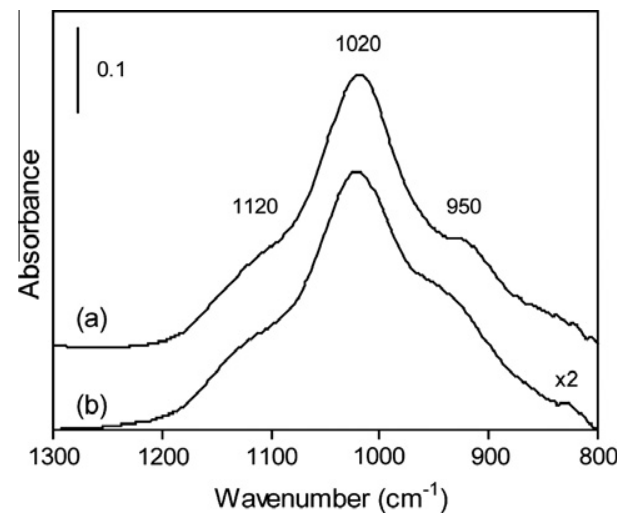

Fig. 6. ATR-FTIR spectra of silicate adsorbed on magnetite at $\mathrm{pH} 8.5$ from a $1 \mathrm{mM}$ aqueous water glass solution for $110 \mathrm{~min}$ (a) and from a $1 \mathrm{mM}$ aqueous sodium metasilicate solution for $150 \mathrm{~min}$ (b). For the sake of clarity, the absorbance of spectrum (b) was multiplied by a factor 2 and spectrum (a) was shifted. 
Table 2

Contact angle of surface modified synthetic magnetite measured by the static sessile drop method.

\begin{tabular}{lll}
\hline Treatment & Contact angle $\left(^{\circ}\right)$ \\
\cline { 2 - 3 } & Test 1 (Potapova et al., 2011) & Test 2 \\
\hline As-synthesized magnetite & $22 \pm 3$ & $20 \pm 3$ \\
$4 \mathrm{mM}$ calcium chloride, $1 \mathrm{~h}$ & $19 \pm 2$ & $15 \pm 4$ \\
$0.04 \mathrm{mM}^{-1}$ madium silicate, $1 \mathrm{~h}$ & $\leqslant 10^{\mathrm{a}}$ & $\leqslant 10^{\mathrm{a}}$ \\
$25 \mathrm{mg} \mathrm{L}^{-1}$ maleic acid ester, $1 \mathrm{~h}$ & $44 \pm 3$ & - \\
$25 \mathrm{mg} \mathrm{L}^{-1}$ Atrac, $1 \mathrm{~h}$ & - & $43 \pm 8$ \\
\hline
\end{tabular}

a The exact value of the contact angle could not be estimated since most of the measurements after silicate adsorption were below detection limit of the instrument $\left(10^{\circ}\right)$.

ions resulted in an increased contact angle of magnetite concentrate, despite the pre-treatment with water glass, due to the activation of the magnetite surface for collector adsorption by calcium ions, in accordance with conclusions drawn from spectroscopy data and reported previously (Potapova et al., 2010b). Adsorption of water glass is expected to result in a better wetting and protect magnetite from collector adsorption, whereas collector adsorption (before bi-layer structure formation) has an opposite effect on wettability. According to Rao (2010), flotation of magnetite fines with Atrac 1563 increases sharply when the contact angle of the magnetite surface is above $70^{\circ}$. In the present work, the surface of the magnetite concentrate had a contact angle of $60^{\circ}$ at the highest concentration of the collector and would probably not be hydrophobic enough to result in the flotation of magnetite. However, hydrophobic areas on the magnetite surface impair wetting of the concentrate and may result in air inclusions inside the green pellets produced by the agglomeration of the magnetite concentrate, which has previously been shown to reduce pellet strength in both wet and dry state (Forsmo et al., 2008).

\subsection{Improvement of magnetite wettability after flotation}

In order to improve the wettability of the flotated magnetite concentrate prior to agglomeration, the effect of two hydrophilizing agents on the contact angle of synthetic magnetite coated with the model collector or Atrac 1563 was investigated. Table 3 shows the contact angle of synthetic magnetite nanoparticles measured by the static sessile drop method before and after adsorption of a hydrophilizing agent.

When a magnetite film treated with a collector was subjected to conditioning with $4 \mathrm{mM} \mathrm{CaCl}_{2}$ and $0.4 \mathrm{mM}$ sodium metasilicate at

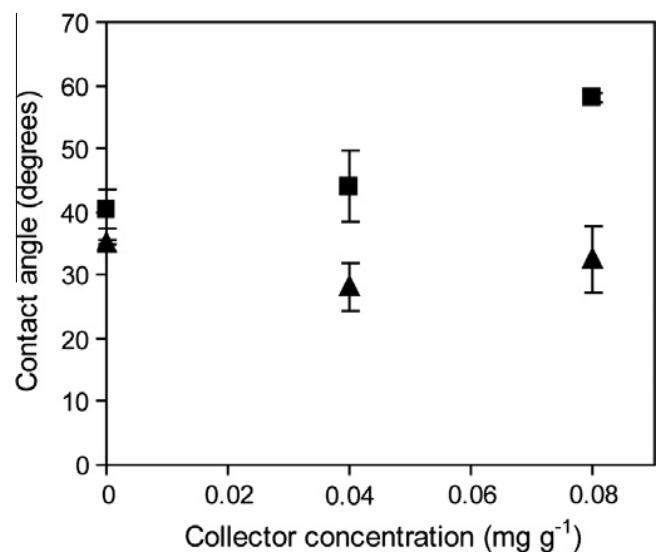

Fig. 7. Water contact angle of magnetite concentrate upon modification of the surface with $1 \mathrm{mg} \mathrm{g}^{-1}$ water glass and Atrac 1563 in $10 \mathrm{mM} \mathrm{NaCl}(\boldsymbol{\Delta})$ and $4 \mathrm{mM}$ $\mathrm{CaCl}_{2}(\boldsymbol{\square})$ solution at pH 9 measured with the Washburn technique.
Table 3

Change in the contact angle of synthetic magnetite pre-treated with calcium chloride sodium metasilicate, and a collector upon adsorption of sodium metasilicate and ammonium polyacrylate.

\begin{tabular}{llll}
\hline \multicolumn{4}{l}{ Contact angle of synthetic magnetite $\left(^{\circ}\right)$} \\
\hline $25 \mathrm{mg} \mathrm{L^{-1 }}$ model collector & $44 \pm 3 \mathrm{mg} \mathrm{L^{-1 }}$ Atrac 1563 \\
$42 \pm 2$ & $49 \pm 3$ & $43 \pm 8$ \\
$0.4 \mathrm{mM}$ sodium & $12.5 \mathrm{mg} \mathrm{L}^{-1}$ & $0.4 \mathrm{mM}$ sodium & $12.5 \mathrm{mg} \mathrm{L}^{-1}$ \\
metasilicate & polyacrylate & metasilicate & polyacrylate $^{\mathrm{b}}$ \\
$21 \pm 1$ & $24 \pm 6$ & $16 \pm 1$ & $20 \pm 6$ \\
\hline
\end{tabular}

a In the presence of $4 \mathrm{mM} \mathrm{CaCl}$.

b Polyacrylate was added to the solution already containing $4 \mathrm{mM} \mathrm{CaCl}_{2}, 0.4 \mathrm{mM}$ $\mathrm{Na}_{2} \mathrm{SiO}_{3}, 25 \mathrm{mg} \mathrm{L}^{-1}$ collector, and $10 \mathrm{mM} \mathrm{NaCl}$.

$\mathrm{pH} 8.5$ for 1 day, the contact angle was lowered to $21 \pm 1^{\circ}$ for the model collector and to $16 \pm 1^{\circ}$ for Atrac 1563 . The increase in surface hydrophilicity was caused by either desorption of the collector due to concentration gradient or replacement of the collector by silicate species.

Conditioning with ammonium polyacrylate was performed by adding the polymer to a solution already containing calcium chloride, sodium metasilicate and a collector (either Atrac 1563 or the model collector) implying that the desorption of the collector due to the change of concentration could not take place. As a result of the treatment, the contact angle of the magnetite film decreased from $44 \pm 3^{\circ}$ to $24 \pm 6^{\circ}$ for the model collector and from $43 \pm 8^{\circ}$ to $20 \pm 6^{\circ}$ for Atrac 1563 . The observed increase in surface hydrophilicity was probably due to masking of the hydrophobic collector species adsorbed on the magnetite surface by hydrophilic polymer molecules (Potapova et al., 2012; Somasundaran and Cleverdon, 1985).

To verify that silicate and polyacrylate could be used for improving magnetite wettability after flotation, adsorption of these compounds was performed on flotated magnetite concentrate at $\mathrm{pH} 9$ and the contact angle of the magnetite concentrate was measured using the Washburn method.

Fig. 8 shows the effect of water glass adsorption on the wettability of the magnetite concentrate.

A contact angle of $57 \pm 5^{\circ}$ was obtained for the magnetite concentrate before water glass adsorption. Such a high value could be due to residues of the collector adsorbed on the surface after flotation, as has been discussed in Section 3.1.

Treatment with water glass clearly decreased the contact angle of the magnetite concentrate, and the hydrophilizing effect improved with increased concentration of water glass. At the highest water glass dosage ( $3 \mathrm{mg}$ per $\mathrm{g}$ magnetite), the resulting contact angle was $28 \pm 3^{\circ}$, which is rather close to the values obtained for synthetic magnetite and sodium metasilicate presented in Table 3.

Sodium polyacrylate was adsorbed on the magnetite concentrate in the presence of calcium ions $\left(4 \mathrm{mM} \mathrm{CaCl}_{2}\right)$. Fig. 9 shows the effect of polymer adsorption on the wettability of the magnetite concentrate. The contact angle of the magnetite after flotation was $52 \pm 1^{\circ}$, which, again, could indicate that the magnetite surface was partly coated by the flotation collector. Upon polymer adsorption from a $0.04 \mathrm{mg} \mathrm{g}^{-1}$ solution, the contact angle of magnetite concentrate decreased; however, the variation of the contact angle within the replicates was in the range $34-54^{\circ}$ indicating an uneven distribution of the polymer on the magnetite surface. Increasing the polymer concentration at a constant concentration of calcium ions did not result in further decrease of the contact angle. A possible explanation could be the depletion of the magnetite surface sites available for polymer adsorption already at lower concentration of the polymer, or the dependence of polymer adsorption on the concentration of calcium ions, i.e. on the calcium-to-polymer ratio. 


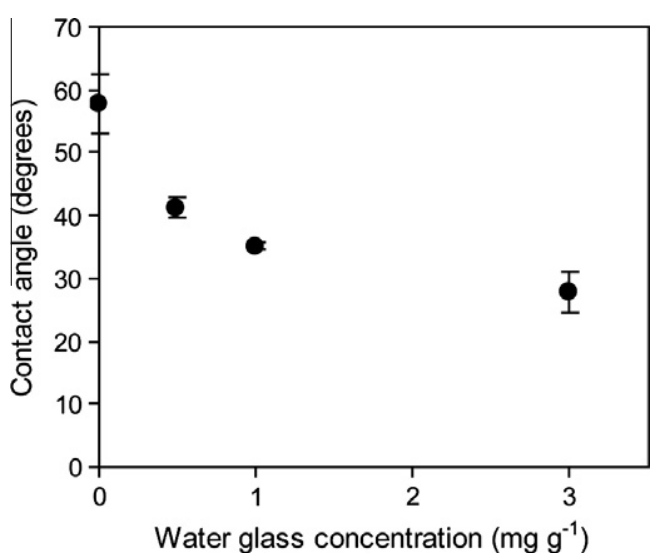

Fig. 8. Water contact angle of the magnetite concentrate upon modification of the surface with water glass in $10 \mathrm{mM} \mathrm{NaCl}$ at $\mathrm{pH} 9$ for $9 \mathrm{~h}$ measured with the Washburn technique. Prior to water glass adsorption, the concentrate was preconditioned with $10 \mathrm{mM} \mathrm{NaCl}$ at $\mathrm{pH} 9$ for $1 \mathrm{~h}$.

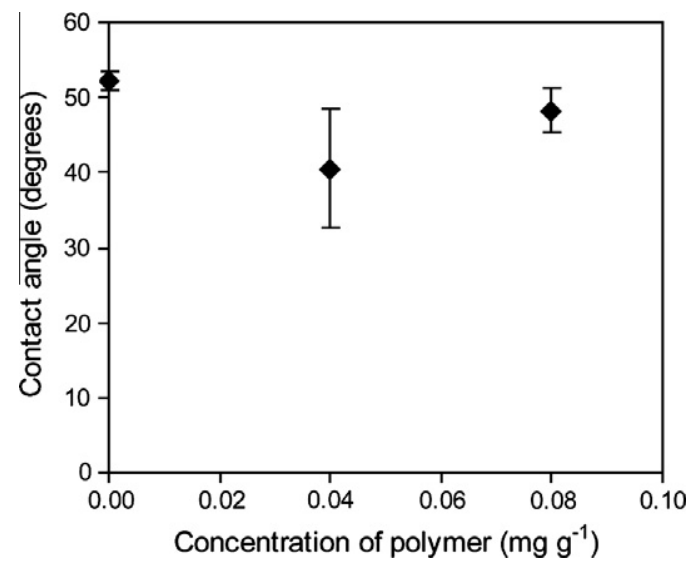

Fig. 9. Water contact angle of the flotated magnetite concentrate upon modification of the surface with sodium polyacrylate in $4 \mathrm{mM} \mathrm{CaCl}_{2}$ at $\mathrm{pH} 9$ for $1 \mathrm{~h}$ measured with the Washburn technique. The point at $0 \mathrm{mg} \mathrm{g}^{-1}$ represents the contact angle of asreceived magnetite.

To test the latter hypothesis, polymer adsorption on magnetite was performed at different concentrations of calcium ions keeping the concentration of the polymer constant at $0.04 \mathrm{mg} \mathrm{g}^{-1}$ magnetite, see Fig. 10. When polymer adsorption was performed without calcium ions, the contact angle of the magnetite concentrate was virtually the same as that of the as-received concentrate suggesting that in the absence of calcium, the polymer was not effective in improving the wettability of the magnetite surface. The slight increase in the measured contact angle (to $55 \pm 1^{\circ}$ ) was possibly caused by desorption of hydrophilic silicate species, which are expected to be present on the magnetite surface after flotation.

When $4 \mathrm{mM}$ calcium chloride was added to the polymer solution, the contact angle of the magnetite concentrate decreased to $41 \pm 8^{\circ}$ as discussed above. Further increase in calcium concentration to $6 \mathrm{mM}$ resulted in an even lower contact angle $\left(25 \pm 11^{\circ}\right)$ confirming the importance of the calcium-to-polymer ratio for polymer adsorption onto magnetite. Since the concentration of calcium in the process water at the LKAB concentrating plant in Kiruna, Sweden, is $8.8 \mathrm{mM}$ (see Table 1), even better effect of polyacrylate adsorption on the wettability of the magnetite concentrate could be expected.

An increased adsorption of polyacrylic acid on aluminium oxide in the presence of calcium ions was reported (Vermöhlen et al., 2000). The authors explained such behaviour by the ability of cal-

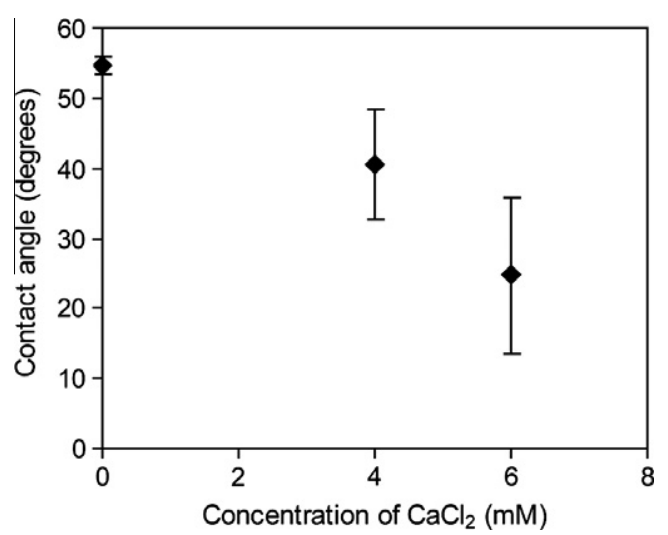

Fig. 10. Water contact angle of the magnetite concentrate upon modification of the surface with $0.04 \mathrm{mg} \mathrm{g}^{-1}$ sodium polyacrylate at $\mathrm{pH} 9$ for $1 \mathrm{~h}$ measured with the Washburn technique. The point at $0 \mathrm{mM}$ represents the contact angle of the magnetite concentrate treated with sodium polyacrylate in the presence of $10 \mathrm{mM}$ $\mathrm{NaCl}$ and no calcium ions.

cium ions to screen the negative charge of the carboxylic groups in the polymer more efficiently as compared to monovalent sodium ions, allowing the polymer to adopt a more coiled conformation on the surface thus increasing surface loading. Hence, when the polymer concentration was increased without increasing the concentration of calcium ions, the amount of calcium ions was not sufficient to facilitate the adsorption of the highly negatively charged polyacrylate onto the magnetite surface also being negatively charged at this $\mathrm{pH}$.

It is important to mention here that the decrease in the contact angle in Fig. 10 is not likely to be due to increased ionic strength upon increasing calcium concentration. According to Vermöhlen et al., polyacrylate adsorption on alumina in the presence of $3.3 \mathrm{mM}$ calcium chloride was twice higher than adsorption from solution containing $10 \mathrm{mM} \mathrm{NaCl}$, having the same ionic strength (Vermöhlen et al., 2000).

The fact that polyacrylate improves the wettability of magnetite more efficiently in the presence of calcium ions makes it suitable for application in processes having a process water rich in calcium ions. Thereby, polyacrylate could be a good candidate for improving the wettability of magnetite after reverse flotation from calcareous gangue minerals.

\section{Conclusions}

In spite of differences in surface properties and morphology of synthetic and natural magnetite particles, similar tendencies were observed for adsorption of calcium ions, soluble silicates, anionic carboxylate surfactants, and polyacrylate polymers on these materials, as illustrated by contact angle measurements. It was confirmed that the wettability of magnetite was reduced by collector adsorption when calcium ions were present in the system, despite pre-conditioning with water glass. Wettability of the flotated magnetite concentrate could be significantly improved by prolonged treatment with water glass or rather short conditioning with sodium polyacrylate, in agreement with the results obtained for the synthetic magnetite nanoparticles. Better wetting of the concentrate would facilitate wet agglomeration and could possibly increase the strength of iron ore pellets produced.

\section{Acknowledgements}

This is a contribution by the Centre of Advanced Mining and Metallurgy (CAMM) at Luleå University of Technology, Sweden. 
The authors acknowledge the financial support from the Hjalmar Lundbohm Research Centre (HLRC). The Knut and Alice Wallenberg Foundation is acknowledged for financial support of the Magellan SEM instrument.

\section{References}

Beentjes, P.C.J., Van Den Brand, J., De Wit, J.H.W., 2006. Interaction of ester and acid groups containing organic compounds with iron oxide surfaces. Journal of Adhesion Science and Technology 20 (1), 1-18.

Dobson, K.D., McQuillan, A.J., 1999. In situ infrared spectroscopic analysis of the adsorption of aliphatic carboxylic acids to $\mathrm{TiO}_{2}, \mathrm{ZrO}_{2}, \mathrm{Al}_{2} \mathrm{O}_{3}$, and $\mathrm{Ta}_{2} \mathrm{O}_{5}$ from aqueous solutions. Spectrochimica Acta - Part A: Molecular and Biomolecular Spectroscopy 55 (7-8), 1395-1405.

Forsmo, S.P.E., 2005. Oxidation of magnetite concentrate powders during storage and drying. International Journal of Mineral Processing 75 (1-2), 135-144.

Forsmo, S.P.E., Forsmo, S.E., Björkman, B.M.T., Samskog, P.O., 2008. Studies on the influence of a flotation collector reagent on iron ore green pellet properties. Powder Technology 182 (3), 444-452.

Fuerstenau, D.W., 2007. A century of developments in the chemistry of flotation processing. In: Fuerstenau, M.C., Jameson, G., Yoon, R.-H. (Eds.), Froth flotation: a century of innovation. Society for Mining, Metallurgy, and Exploration, Inc., Littleton, pp. 3-64.

Galindo-González, C., De Vicente, J., Ramos-Tejada, M.M., López-López, M.T. González-Caballero, F., Durán, J.D.G., 2005. Preparation and sedimentation behavior in magnetic fields of magnetite-covered clay particles. Langmuir 21 (10), 4410-4419.

Gustafsson, J.O., Adolfsson, G., 1997. Adsorption of carboxylate collectors on magnetite and their influence on the pelletizing process. In: International, X.X. (Ed.), Mineral Processing Congress, GDMB Gessellschaft fur Bergbau, Metallurgie, Rohstoff- und Umwelttechnik. Clausthal-Zellerfeld, pp. 377390 .

Holmberg, K., Jönsson, B., Kronberg, B., Lindman, B., 2003. Surfactants and Polymers in Aqueous Solution, second ed. John Wiley \& Sons Ltd., Chichester.

Hwang, Y.S., Lenhart, J.J., 2008. Adsorption of C4-dicarboxylic acids at the hematite/ water interface. Langmuir 24 (24), 13934-13943.

Iwasaki, I., Zetterström, J.D., Kalar, E.M., 1967. Removal of fatty acid coatings from iron oxide surfaces and its effect on the duplex flotation process and on pelletizing. Transaction Society of Mining Engineers AIME 238, 304-312.

Kirchberg, S., Abdin, Y., Ziegmann, G., 2011. Influence of particle shape and size on the wetting behavior of soft magnetic micropowders. Powder Technology 207 (1-3), 311-317.
Odman, F., Ruth, T., Ponter, C., 1999. Validation of a field filtration technique for characterization of suspended particulate matter from freshwater. Part I. Major elements. Applied Geochemistry 14 (3), 301-317.

Potapova, E., Carabante, I., Grahn, M., Holmgren, A., Hedlund, J., 2010a. Studies of collector adsorption on iron oxides by in situ ATR-FTIR spectroscopy. Industrial and Engineering Chemistry Research 49 (4), 1493-1502.

Potapova, E., Grahn, M., Holmgren, A., Hedlund, J., 2010b. The effect of calcium ions and sodium silicate on the adsorption of a model anionic flotation collector on magnetite studied by ATR-FTIR spectroscopy. Journal of Colloid and Interface Science 345 (1), 96-102.

Potapova, E., Yang, X., Grahn, M., Holmgren, A., Forsmo, S.P.E., Fredriksson, A., Hedlund, J., 2011. The effect of calcium ions, sodium silicate and surfactant on charge and wettability of magnetite. Colloids and Surfaces A 386, 79-86.

Potapova, E., Grahn, M., Holmgren, A., Hedlund, J., 2012. The effect of polymer adsorption on the wetting properties of partially hydrophobized magnetite. Journal of Colloid and Interface Science. 367 (1), 478-484.

Qiu, G., Jiang, T., Fa, K., Zhu, D., Wang, D., 2004. Interfacial characterizations of iron ore concentrates affected by binders. Powder Technology 139 (1), 1-6.

Rao, K.H., 2010. Role of non-ionic surfactant in fatty acid phosphate gangue flotation from magnetite fines. In Conference in Minerals Engineering. Luleå University of Technology, Luleå, Sweden, pp. 169-183.

Shang, J., Flury, M., Harsh, J.B., Zollars, R.L., 2008. Comparison of different methods to measure contact angles of soil colloids. Journal of Colloid and Interface Science 328 (2), 299-307.

Smart, R.S.C., Skinner, W., Gerson, A.R., Mielczarski, J., Chryssoulis, S., Pratt, A.R., Lastra, R., Hope, G.A., Wang, X., Fa, K., Miller, J.D., 2007. Surface characterization and new tools for research. In: Fuerstenau, M.C., Jameson, G., Yoon, R.-H. (Eds.), Froth Flotation: a Century of Innovation, Society for Mining, Metallurgy, and Exploration, Inc., Littleton, pp. 283-338.

Somasundaran, P., Cleverdon, J., 1985. A study of polymer/surfactant interaction at the mineral/solution interface. Colloids and Surfaces 13 (C), 73-85.

Wang, Y., Ren, J., 2005. The flotation of quartz from iron minerals with a combined quaternary ammonium salt. International Journal of Mineral Processing 77 (2), $116-122$.

Vermöhlen, K., Lewandowski, H., Narres, H.D., Schwuger, M.J., 2000. Adsorption of polyelectrolytes onto oxides - The influence of ionic strength, molar mass, and $\mathrm{Ca}^{2+}$ ions. Colloids and Surfaces A: Physicochemical and Engineering Aspects 163 (1), 45-53.

Yang, X., Roonasi, P., Holmgren, A., 2008. A study of sodium silicate in aqueous solution and sorbed by synthetic magnetite using in situ ATR-FTIR spectroscopy. Journal of Colloid and Interface Science 328 (1), 41-47.

Yang, X., Roonasi, P., Jolsterå, R., Holmgren, A., 2009. Kinetics of silicate sorption on magnetite and maghemite: an in situ ATR-FTIR study. Colloids and Surfaces A $343(1-3), 24-29$. 